\title{
Student reasoning in hydrodynamics: Bernoulli's principle versus the continuity equation
}

\author{
Claudia Schäfle@ ${ }^{*}$ \\ Faculty of Applied Natural Sciences and Humanities, \\ Rosenheim Technical University of Applied Sciences, 83024 Rosenheim, Germany \\ Christian Kautz $(1)$ \\ Engineering Education Research Group, Hamburg University of Technology, 21073 Hamburg, Germany
}

(Received 8 May 2020; revised 5 April 2021; accepted 4 May 2021; published 29 June 2021)

\begin{abstract}
We report on an investigation of student thinking about steady-state pipe flow of an incompressible fluid. About 250 undergraduate engineering students were given a test consisting of two hydrodynamics questions, combining multiple-choice format with subsequent open-ended explanations. There is substantial evidence that students have difficulty applying and prioritizing the two basic principles of mass conservation (expressed in the continuity equation) and energy conservation (i.e., Bernoulli's equation). When faced with questions that involve gravity, dissipative effects ("friction"), or a visible pressure drop, a considerable number of students did not invoke the continuity equation in situations where applying it is a necessary step for arriving at the correct answer. Instead, even after lecture instruction on this topic, many of the first-year students based their answers on ill-supported assumptions about local pressures. Some of them used formal arguments from a simplified Bernoulli equation ("lower pressure means higher velocity"), while others based their answer on intuitive arguments ("higher pressure leads to higher velocity"). We also found reasoning based on analogies to single-particle motion ("flow velocity decreases when flowing upwards or friction is present"). Contrary to other researchers, we did not see any evidence for the hypothesis that students think of water as a compressible fluid. Instead, students' answers often indicate a lack of understanding of the conservation of mass or its implications for incompressible fluids or of the role that this principle plays in the context of fluid flow. In addition, our data indicate that some students have more general difficulties in describing and reasoning about technical situations, such as applying equations containing multiple variables, distinguishing spatial differences in a quantity from its changes with respect to time, or realizing the meaning of idealizations. We also present some evidence that different levels of activation of students during instruction influence the prevalence of these difficulties and discuss some implications for instruction.
\end{abstract}

DOI: 10.1103/PhysRevPhysEducRes.17.010147

\section{INTRODUCTION}

The phenomenon of a steady pipe flow of an incompressible fluid plays an important role in many natural and technical systems and is therefore taught early on in many university science and engineering degree programs. The topic, sometimes referred to as hydrodynamics, may be covered as part of an introductory physics course or be included in an engineering mechanics or engineering thermodynamics course and, at this level, usually includes two fundamental principles, the conservation of mass and of energy. For engineering students, a more detailed

\footnotetext{
*claudia.schaefle@th-rosenheim.de
}

Published by the American Physical Society under the terms of the Creative Commons Attribution 4.0 International license. Further distribution of this work must maintain attribution to the author(s) and the published article's title, journal citation, and DOI. description of the behavior of fluids is often taught at a later stage in an entire course devoted to fluid mechanics.

The flow of incompressible fluids, in particular Bernoulli's equation, has received considerable attention in the physics and engineering education literature. However, many of these studies are devoted to innovative approaches in teaching fluid flow. Some more recent examples of this type of work are given in Refs. [1-3]. A shift in focus towards students ideas about the topic can be seen in the development and implementation of two multiple-choice instruments (concept inventories) during the past two decades [4-7]. Most recently, two studies on misconceptions or conceptual difficulties in hydrodynamics were published $[8,9]$. The latter of these two publications also cites one further study on this topic that is currently published only in Spanish.

Specifically, Brown et al. carried out interviews with second-, third-, and fourth-year engineering students and observed that students think differently about horizontal 
and vertical pipelines [8]. The authors explain this "dramatic shift in the participants' conceptual understanding" by invoking "framework theory," a particular type of conceptual change theory. Suarez et al. used written tests and interviews to identify various misconceptions in the context of ideal (i.e., nonviscous) fluid flow among students in a general physics course for prospective engineers [9]. The authors of that study also observe that students incorrectly generalize results from hydrostatics and that they "faced difficulties to understand how different parts of a fluid in motion interact, failing to link kinematics with dynamics."

Both studies, however, focus largely on the students' ability to predict and explain changes in pressure and to apply mainly one of the two key principles, namely, conservation of energy, in the form of Bernoulli's equation. Student answers in interviews and on written tests that seem problematic in light of the other, more basic principle, i.e., conservation of mass, are interpreted by these researchers as a consequence of the students' idea that water is compressible.

Informal observations of students at a University of Applied Sciences in Germany initially led us to a different hypothesis, namely, that students have difficulty distinguishing between and understanding the relationship of these two principles and, consequently but unjustifiably, focus on Bernoulli's principle alone. We therefore saw a need for a more detailed investigation of student understanding of the principle of conservation of mass in the context of incompressible fluids, as expressed in the continuity equation. Moreover, research on student difficulties with a similar idea in the context of electric circuits [10], i.e., the conservation of charge as expressed in Kirchhoff's current law, motivated us to probe more deeply to what extent introductory-level students are able to consistently apply the principle of conservation of mass. In order to do so, we gave an ungraded written test to a total of about 200 students in different first-year physics courses and to about 50 students in their second or third year in various sevensemester engineering bachelor's degree programs.

Some parts of this study have been reported before $[11,12]$. In this paper, we briefly describe the first-year and upper-level groups and the instruction that they had received (Sec. IV), outline the data analysis process that we followed (Sec. V), describe the test and the distribution of student answers (Sec. VI) as well as our analysis of student reasoning (Sec. VII). Finally, we present some implications for the teaching of hydrodynamics (Sec. VIII) before presenting a summary and conclusion of our entire study. We begin by describing our methodological framework (Sec. II) followed by a brief description of the relevant course content and our research question (Sec. III).

\section{METHODOLOGICAL FRAMEWORK}

For several decades, researchers in science, technology, engineering, and mathematics education have observed that students often hold on to their own ideas about mechanisms in the physical and technical realm that they have constructed based on their prior experience in everyday life. These ideas can be erroneous or incomplete, and are sometimes referred to as "misconceptions." When asked to make qualitative predictions or to solve problems, students will make use of these ideas and may therefore fail to arrive at a correct and complete answer [13].

As mental processes cannot be observed directly, student ideas or, on a larger scale, their mental models have been inferred from the students' observed behavior, including the answers they choose and the explanations they give to support these answers. Different ways of categorizing student thinking are therefore possible and have been proposed. Hammer, for example, emphasizes "conceptual resources" that students draw on and that may be more or less appropriate or helpful for the particular problem they are asked to solve [14]. DiSessa stipulates that there exist some most basic units among such conceptual resources and refers to these as $p$-prims [15], while Minstrell has referred to such conceptual units as "facets" of students' knowledge [16].

In this work, we take a perspective similar to that described by Heron, identifying "student difficulties [...] that must be addressed in instruction" [17] for students to gain a functional understanding of the subject matter, that is (as defined by McDermott) "the ability to interpret and use knowledge in situations different from those in which it was initially acquired" [18]. The identification of such difficulties then serves as an empirical foundation for the development of instructional materials that foster this kind of understanding.

While our aim here is not (primarily) to advance the theoretical framework to model student thinking, we note that student difficulties observed in one area often parallel those observed in another. Thus, we take our findings in the context of hydrodynamics as a starting point to identify general patterns of student thinking.

\section{TECHNICAL BACKGROUND AND RESEARCH QUESTIONS}

One of the intended learning outcomes of the hydrodynamics unit in the introductory physics courses considered here may be stated as follows: Students demonstrate their understanding of the following concepts by applying them correctly to various problems:

(1) Continuity equation (conservation of mass):

For a steady flow of constant density in a pipe (without sinks and sources) the volume flow $\dot{V}$ at two arbitrarily chosen positions along the pipe is the same at any given time:

$$
\dot{V}=A \cdot \bar{v}=c \quad \text { or } \quad A_{1} \cdot \bar{v}_{1}=A_{2} \cdot \bar{v}_{2} .
$$

Here, $A_{i}$ and $\bar{v}_{i}$ denote the cross-sectional area and the mean velocity (averaged over the cross-sectional 
area) of the fluid at position 1 and 2, respectively, and $c$ is a constant (i.e., independent of position).

(2) Bernoulli's equation with pressure losses (workenergy principle including dissipative losses):

In a steady, incompressible pipe flow the sum of all energy forms per volume, i.e., the sum of static pressure (or flow work per volume), kinetic energy density, and potential energy density is the same along the pipe if losses can be neglected. In the case of real flows, losses are taken into account by adding a term that represents the pressure loss between position 1 and 2 (where position 2 is assumed to be downstream from position 1):

$$
\begin{aligned}
p_{1} & +\frac{1}{2} \rho \cdot \bar{v}_{1}^{2}+\rho g h_{1} \\
& =p_{2}+\frac{1}{2} \rho \cdot \bar{v}_{2}^{2}+\rho g h_{2}+\Delta p_{\operatorname{diss} 12} .
\end{aligned}
$$

A typical application of these two concepts, often presented in textbooks or lectures, is the Venturi nozzle, which can be used to measure the volume flow in a pipe [19].

Of the two concepts, the principle of continuity (or conservation of mass) may be seen as more basic as it concerns only the kinematics of the flow. Only Bernoulli's principle introduces a physical quantity, the pressure, that can be thought of as causing changes in the fluid's motion. Based on our observations of student thinking in other branches of physics, e.g., single-particle motion, we suspect that many students treat concepts in kinematics as obvious and consequently focus their attention almost exclusively on the dynamics, thereby neglecting that some results about a given problem can or even must be inferred from the concept of continuity. These thoughts led us to the following research question for this study:

To what extent are students in an engineering degree program at a German University of Applied Sciences able to apply the principle of conservation of mass (as expressed by the continuity equation) to simple situations of incompressible fluid flow? In particular, is their ability to apply this principle sufficiently robust for them to successfully do so even in the presence of distracting factors in the problem setting, such as the influence of gravity, dissipative effects (commonly referred to as "friction") or a visible pressure drop. Finally, what patterns of reasoning do students display in this context?

\section{APPROACH AND CONTEXT OF INVESTIGATION}

In order to investigate students' ideas in the context of basic principles in pipe flow we developed three questions that each combine multiple choice and subsequent freeresponse formats asking for student reasoning. We expected all three questions to reveal interesting ideas, but were worried about possible interference between two of them when given to the same students. Consequently, we combined the first question with the second or the third, respectively, to form a short written test. Approximately half of the students in each group were randomly assigned either of the two versions of the test. All questions will be described in Sec. VI and shown in the Appendix.

The test was administered to undergraduate (i.e., bachelor's) students in their first year (groups 1-5, 197 students) or in their second or third year (group 6, 51 students) in different engineering and technology programs at the Rosenheim Technical University of Applied Sciences (Germany) in the academic year 2014-2015. This institution, like many other Universities of Applied Sciences in Germany, offers various seven-semester bachelor's degrees that aim to prepare students for engineering careers in industry or subsequent studies towards a master's degree. The focus of these programs is on applying science to industrial research and development, not on academic research. The scopes of the physics courses in different degree programs are very similar, resulting in mutual accreditation in case of a student changing their major. There is some variation in students' backgrounds and level of preparation, but differences between cohorts tend to be greater than those between degree programs. In the particular year of our investigation, the averages of the FCI ([20]) pretest scores in the three programs were $10.4 \pm 4.7$ (group 2), $10.7 \pm 4.9$ (group $1+$ group 5), and $13.7 \pm 5.8$ (group $3+$ group 4 ). As group 6 began their studies one year before, there are no pretest scores available for these students. We have no indication of any systematic differences in the students' motivation between different degree programs.

Relevant details about the various groups and the respective numbers of students in the study are summarized in Table I.

The groups had been taught by different lecturers using different methods of teaching: some in a traditional lecture format, others in interactive formats combining Just-inTime Teaching (JiTT) [21] and Peer Instruction (PI) [22]. The groups also differed with respect to the type and amount of instruction that had been completed at the time of the test. One group was given the test after self-study in preparation of a lab experiment before any lecture instruction on pipe flow (group 1), others after relevant lecture instruction only (group 2) or after lecture and a corresponding lab experiment (groups 3, 4, and 5). In the three-hour laboratory experiment, the respective students investigated the pressure loss of steady water flow in a straight, horizontal pipe with constant diameter. Group 6 had completed the entire course including the final examination one or two years prior to taking the test. Additionally, group 6 had in the meantime attended further courses like thermodynamics and fluid mechanics. 
TABLE I. List of populations (groups) involved in the study and relevant details, including timing of the test, instructional method, and number of participants in each group. JITT, PI, and lab denote Just-in-Time Teaching, Peer Instruction, and a corresponding laboratory experiment as described in Sec. IV. The last three columns refer to the numbers of students answering the corresponding question (IP = inclined-pipe question; HP = horizontal-pipe question; MP = manometer-pipe question; see Sec. VI).

\begin{tabular}{lcccccc}
\hline \hline Group & Prior instruction & Degree program and semester & Instructional method and timing of test & $N(\mathrm{IP})$ & $N(\mathrm{HP})$ & $N(\mathrm{MP})$ \\
\hline 1 & First year, & Energy \& Building & Autonomous study before lab; & 41 & 20 & 21 \\
& self-study & Technology 1 & no lecture prior to test & & & \\
2 & First year, & Interior Engineering 1 & After traditional lecture; no lab & 53 & 28 & 25 \\
3 & postlecture & Wood Technology 1B & After traditional lecture + lab & 32 & 17 & 15 \\
4 & & Wood Technology 1A & After lecture with JITT\&PI + lab & 32 & 17 & 15 \\
5 & & Energy \& Building Technology 1 & After lecture with JITT\&PI + lab & 39 & 22 & 17 \\
6 & 2nd or 3rd year & Energy \& Building & One or two years after & 51 & 24 & 27 \\
& & Technology 3,5 & relevant lecture + lab & & & \\
\hline \hline
\end{tabular}

While students were given no strict time limit for completing the test, most of them finished within about $5 \mathrm{~min}$. The test was closed book and students were not given any equations as part of the test. $100 \%$ of the test sheets were returned, i.e., all students present in class on the day of the test participated in the study. The students' answers were neither graded nor returned, but students were invited to ask questions about the test.

\section{DATA ANALYSIS METHODS}

Data analysis proceeded in two stages, a quantitative analysis of student answers and explanations, and a more detailed qualitative analysis of student reasoning. In the quantitative analysis, student answers to the multiplechoice questions contained in each version of the test were first counted as correct or incorrect. ${ }^{1}$ Subsequently, student explanations for their answers were rated as "correct," "incorrect," or "unclear or missing" according to whether the relevant physical principle was applied. (A detailed description of the sorting criteria is given in Sec. VI.)

As, ultimately, our intention is to help instructors in hydrodynamics gain insight into student thinking and to design instructional materials, we complemented the quantitative study with a qualitative analysis (Sec. VII). After an initial perusal of all student explanations, we jointly identified for each question an initial set of four to six ideas invoked by the students to support their answers. In a second round, each student answer was coded according to the predominant idea, noting that some explanations used more than one idea and a few could not be classified at all. The third step of the analysis aimed at characterizing each student by their conception of fluid flow according to the ideas used in the entire test. Because of the inconsistency of student ideas across different questions, this proved to be

\footnotetext{
${ }^{1}$ The horizontal-pipe question, part $b$, while not strictly in multiple-choice format, asks for an answer of yes or no and was therefore treated as multiple choice.
}

not useful for our goal. In a concluding step, we therefore focused on the assumptions used and the inferences drawn by the students, generalizing these to identify characteristic patterns of student reasoning.

\section{TEST AND OVERVIEW OF QUANTITATIVE RESULTS}

In all three questions, situations are described in which water flows out of a water tank through straight pipes of uniform cross section (Fig. 1). The water level of the tank is kept constant by a feedback mechanism (not shown). The students are asked if the velocity in the center of the pipe cross section at position 2 (located further downstream) is greater than, less than, or equal to that at position 1 and to explain their answer. In the first question, subsequently referred to as the inclined-pipe question (IP), the pipe is inclined upward. In the second question, the pipe is horizontal [horizontal-pipe question (HP)]. Here the students are first told to assume that friction can be neglected (part a), and afterwards asked if their answer would change (and why) if friction was to be taken into account (part b). In the third question, the pipe is again horizontal and three manometers are shown with their water levels decreasing along the flow [manometer-pipe question (MP)]. In addition students are asked to explain the water levels in the manometer tubes (if they had not done so before). A translation of the test into English is provided in the Appendix.

With these questions our intent was to probe whether the students' understanding of the concept of continuity is stable or whether it would break down in situations that involve gravity (IP), that explicitly take into account dissipation (HP) or that provide information about a pressure drop along the pipe (MP).

For all three questions, applying the continuity equation [Eq. (1)] in the case of constant cross section leads to the answer that the velocity is the same at both positions. We counted students' explanations as correct if they either 


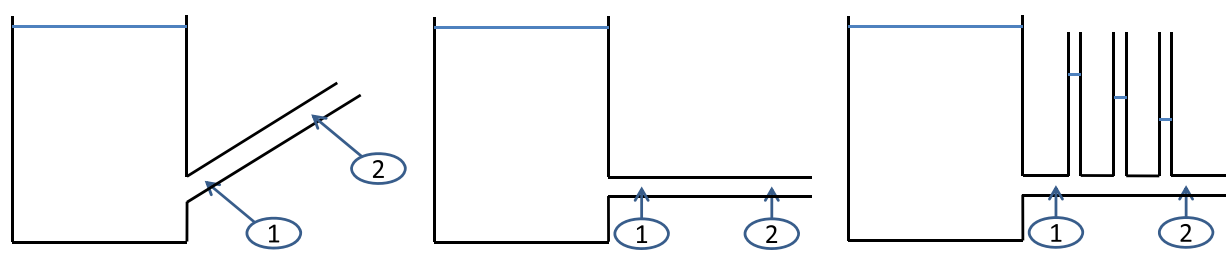

FIG. 1. From left to right: sketch of inclined-pipe, horizontal-pipe, and manometer-pipe question.
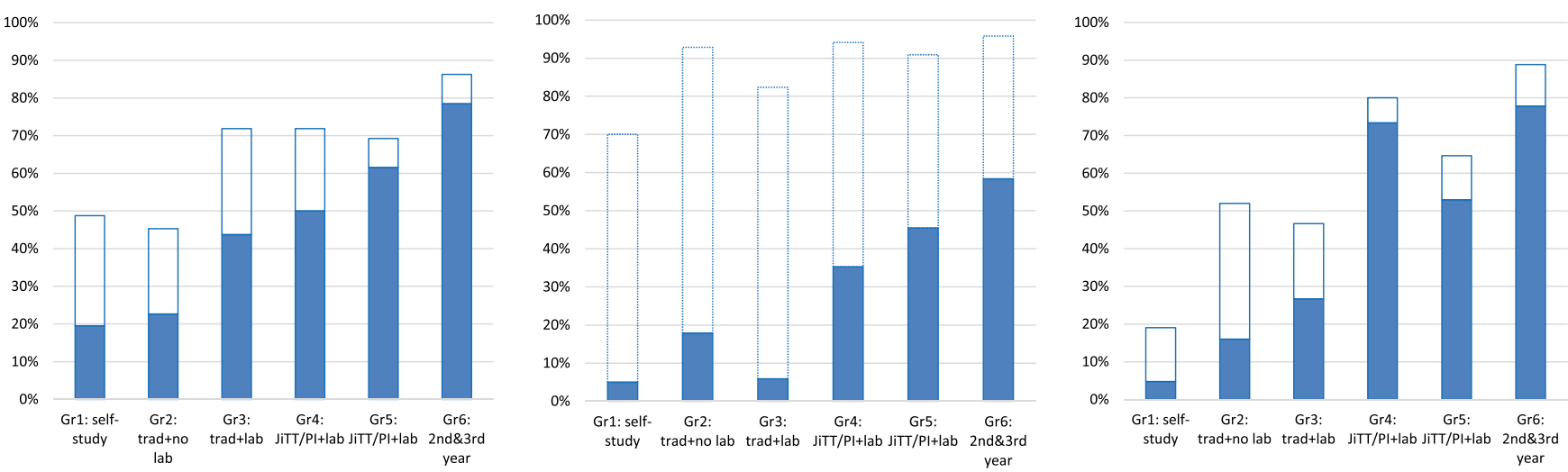

FIG. 2. From left to right: Results of IP, HP, and MP question for each student group (details of the groups see Table I). For IP and MP the empty bars show the percentages of correct answers and the solid bars show the fraction of students giving correct reasoning. For HP the empty bars with dashed frame indicate the percentages of correct answers to the case, when friction is neglected, while the solid bars display percentages of students with correct reasoning stating that friction has no influence on their answer.

showed this reasoning in detail or gave similar but somewhat incomplete reasoning, e.g., simply referring to the uniform cross section or to conservation of mass. Moreover, we counted explanations for HP and MP as correct if the students only referred to their (correct) explanation regarding IP.

The results of all three questions are presented in Fig. 2, with percentages of correct answers (regardless of reasoning) and correct reasoning (in the sense defined above) both shown.

Inspecting those results, we observe the following:

- The percentages of correct answers and reasoning vary strongly across the six groups, with the self-study group (group 1) generally showing the lowest and the second and third year students (group 6) the highest values.

- Among the first-year students who had received instruction (groups 2 to 5) the teaching format seems to matter considerably, with interactive formats (groups 4 and 5) achieving notably better results.

- The fraction of students supporting their answer with correct reasoning (solid bars) is often substantially lower than the fraction of students giving correct answers regardless of reasoning (empty bars). ${ }^{2}$

\footnotetext{
${ }^{2}$ Note, however, that in the case of HP (center diagram) the empty bars refer to the case without friction.
}

- More specifically, the maximum percentage among the groups of first-year students who applied the concept correctly was

(i) about $62 \%$ when the situation involved gravity (IP),

(ii) about $45 \%$ when the situation explicitely involved friction (HP), and

(iii) about $73 \%$ when the situation involved a visible pressure drop (MP).

These observations lead us to conclude that even in the strongest groups many students have difficulties applying the principle of continuity correctly, especially in situations that involve friction. Instruction in hydrodynamics needs to specifically address these difficulties. As a first step, in this paper, we analyze student thinking about fluid flow in the context of the above problems in detail.

\section{ANALYSIS OF STUDENT REASONING}

The majority of the students who used the idea of uniform mass flow in their explanations also arrived at the correct answer, stating that the velocity of the water is the same at the locations considered. Students who did not base their answers on this principle used other types of reasoning that often indicated specific misconceptions about fluid flow, in particular about the relationship between pressure and flow velocity. In this section, we discuss common types of reasoning, illustrate them with sample answers and provide 
estimates of their prevalence where possible. However this is not intended as a quantitative study as incidence rates vary by group and by question.

\section{A. Failure to use (or lack of confidence in) the continuity equation}

In all groups within our study, a considerable fraction of the students did not invoke the continuity equation in one or both of the questions posed. After lecture instruction in the first-year courses, those percentages ranged from onequarter to three-quarters. In the majority of cases, most likely, this is not due to a failure to remember the equation. Instead, students' answers often indicate a lack of understanding of the conservation of mass or its implications for incompressible fluids, or of the role that this principle plays in the context of fluid flow. As an example, we quote a firstyear student after lecture with active learning methods who explicitly invokes the conservation of mass but then bases his answers on the observed change in pressure.

MP a): [greater] Conservation of mass: What is fed in must come out again $\rightarrow$ the static pressure decreases. Consequently, the dynamic pressure increases. (Student $\left.716,{ }^{3}\right)$

The above interpretation is supported by the relatively large number of students who changed their initial answers, including some who moved from a correct to an incorrect answer. The following two quotes, one from a thirdsemester student who changed their answer (to IP) from correct to incorrect and then to correct again, the other from a first-semester student changing their answer (to MP) from correct to incorrect illustrate the distracting effect of ideas about pressure:

IP: [same] Since the cross section of the pipe stays the same, the velocity stays the same.

[tess] Becatuse of the difference in height, the pressure is ehanging and hence the velocity.

[same] Cross section stays the same $\rightarrow$ velocity stays the same. (Student 107)

$M P$ a): [same] The same amount of water that flows in must flew out, therefore $v$ is the same.

[less] From the manometer tubes one can tell the decreasing dynamic pressure $\rightarrow$ which includes the flow velocity. (Student 281)

Even students arriving at the correct answer by considering the uniform pipe cross section-a reasonable starting point for an argument invoking continuity-often did so in logically inconsistent ways by unnecessarily considering

\footnotetext{
${ }^{3}$ This student is part of a later but comparable cohort that was not included in the quantitative analysis above.
}

pressure as part of their argument, as the following quote indicates. ${ }^{4}$

IP: [same] Since the cross section of the pipe is constant, the pressure is the same everywhere and therefore the velocity as well. (Student 315)

By accident, some students who seem to invoke the continuity equation refer to it as the Bernoulli equation:

IP: [same] Because of the same diameter at position 1 and $2 \rightarrow$ Bernoulli equation. (Student 307)

Our result concerning students' failure to use the principle of continuity is in some contrast to findings reported in the literature. Brown et al. report generally higher percentages of students using the idea of continuity in interviews involving pipes with changing diameter (after instruction) [8]. Suarez et al. [9] cite some student quotes from interviews that may suggest difficulties with the principle of continuity but do not probe student thinking in this respect more deeply.

\section{B. Inappropriate use of Bernoulli's principle}

In their answers to question IP, about a quarter of all firstsemester students after lecture (groups 2 through 5) based their answers on correct or incorrect assumptions about the pressures at the respective points and arrived at various conclusions about the velocity at position 2 relative to position 1. Many of these made explicit or implicit use of Bernoulli's equation, thereby concluding that the flow velocity increases or that it decreases along the upwardly inclined pipe, or that it stays the same.

The following answer given by a second-year student is a typical example of an explicit reference to Bernoulli's equation (even if it contains a typographical error):

IP: [less] Since by Bernoulli's equation, the pressure is constant $\left(\frac{1}{2} p v^{2}[\mathrm{sic}]+p+\rho g h=\mathrm{const}\right)$ and the hydrostatic pressure at position 2 is greater than at position 1, the velocity $v$ at position 2 must be smaller than at position 1. (Student 133)

Based on the correct assumption that the potential energy per unit volume (here incorrectly referred to as hydrostatic pressure) at position 2 is greater than at position 1, the student concludes that the velocity at position 2 must be smaller. The student thereby neglects the dependence of the (static) pressure $p$ on altitude and arrives at an incorrect conclusion.

An implicit use of Bernoulli's equation is also very common. As the following two sample answers to IP

\footnotetext{
${ }^{4}$ For the purpose of our categorization in Sec. VI, such reasoning was considered correct if the constant cross section served as a starting point or predominant aspect of the reasoning chain.
} 
illustrate, many students using this type of reasoning not only approach the problem with the wrong principle; they also seem to have difficulty identifying and interpreting the terms in Bernoulli's equation in a correct way.

IP: [less] The velocity at position 2 in the pipe is less than at position 1 since the static pressure increases with height. (Student 257)

IP: [greater] The velocity increases since the opposing static pressure decreases. (Student 208)

In both answers, the respective student infers an increase or decrease in velocity from an opposite change in static pressure, along the lines of an incomplete Bernoulli principle with only two terms. The first student incorrectly assumes that the pressure increases with height (thereby confusing static pressure with the potential energy term, as we will discuss in Sec. VIIE3). The second student correctly assumes a decrease in static pressure but ignores the potential energy term. The specific wording suggests that ideas about a direction of pressure may play a role in arriving at this answer (see also Sec. VII H).

As we illustrate in Sec. VII C, there are also other ways of reasoning that tend to lead students toward concluding that the flow velocity is smaller at position 2 (as compared to position 1). While the above answers might therefore be explained by a general tendency of students to put forth any argument that confirms their preconceived answer (as suggested, for example, by dual-process theories), it is worth noting that the type of reasoning shown here leads similarily often to the conclusion that the water flows faster at position 2 as that it flows more slowly, as the following quote illustrates.

IP: [greater] Since the pressure drops, according to Bernoulli the velocity must increase. (Student 37)

Similar reasoning can be found in students answers to question MP, as the following quotes show:

MP a): [greater] In the water-filled tubes we see that the static pressure inside the pipe becomes smaller, therefore the dynamic pressure must have increased (because there is no slope present). (Student 351)

MP a): [greater] The velocity of the fluid becomes greater since, as a result of the increasing dynamic pressure, the static pressure decreases (see water level in the tubes). (Student 208)

The reader may note that the second of these students used the same line of reasoning in their answer to IP (quoted above). This was also the case for the first student and serves as a further indication that the students' answers are not ex-post rationalizations of a preconceived answer.

One further sample answer may provide additional insight into student reasoning about this situation:
MP a): [greater] [velocity] increases. At greater velocities, the pressure in the tubes decreases. (Student 218)

This student (who answered question IP correctly, although with unclear reasoning) seems to remember the standard illustration of Bernoulli's principle, where for a horizontal flow of varying cross section, manometer tubes at the narrower positions indicate lower pressure as a result of the increased flow velocity.

Finally, we present one quote from a first-semester student in group 2, who uses Bernoulli's principle to arrive at the result that the flow velocity can increase in a pipe of constant diameter if a pressure drop occurs, thereby explicitly contradicting the continuity equation.

MP a): [greater] The dynamic pressure increases, therefore also the velocity. The geodetic pressure [potential energy per volume] can be neglected, the static pressure decreases. MP b): With increasing velocity the pressure in a pipe of constant diameter decreases. (Student 305)

As we have seen, the inverse (although not inversely proportional) relationship between pressure and flow velocity (under certain conditions) that is expressed by Bernoulli's principle, seems to be remembered by many students after initial exposure to the material. However, students often have difficulties associating features of a problem description with the correct terms in the equation, tend to neglect certain terms in that equation (possibly more often the ones they find difficult to interpret) or fail to see the limitations of its applicability. Given the result from previous studies that students have difficulty understanding hydrostatic pressure in fluids at rest [23], this is not surprising.

However, comparing our results to those of other studies on student understanding of hydrodynamics, we point out that students' difficulties with Bernoulli's equation consist not only in (mis)using it in situations in which it does not hold (as was emphasized, for example, by Suarez et al. [9]). An even more severe difficulty (in our opinion) is that students tend to apply it to tasks in which there is not enough information given for a direct application and the continuity equation therefore needs to be applied first.

\section{Incorrect association between pressure and velocity}

Contrary to the reasoning based on a "simplified" Bernoulli's principle shown above (Sec. VII B), other students tend to assume a direct (i.e., local and immediate) causal relationship between the quantities pressure and flow velocity, i.e., they associate lower pressure with smaller velocity and higher pressure with greater velocity at the respective position.

Unlike in the case of reasoning based on Bernoulli's equation, students tend to use this type of reasoning mostly in cases where they perceive a decrease in pressure along 
the flow. This is illustrated by the following answers to question IP, HP, and MP:

IP: [less] Since the pressure decreases, the velocity also decreases. (Student 65)

HP a): [less] The static pressure decreases along the pipe. Therefore the velocity at position 2 is smaller than at position 1. (Student 219)

MP a): [less] As a result of the pressure drop, the velocity of the water decreases in the center of the pipe at position 2. (Student 6)

These answers indicate that students view pressure as a cause of velocity in fluid motion. The answer of student 65, in particular, suggests the presence of this belief, similar to the common (incorrect) idea that a force is necessary to sustain the motion of a point particle.

This intuitive concept of pressure as a cause of velocity in fluid flow has previously been reported in the literature. Our observations are consistent with those of Barbosa (as quoted in Suarez et al. [9]) and Brown et al. [8] (see, e.g., student 106 in that work). Similarly, Chi et al. [24] report that medical students state that "no pressure implies no flow."

In summary, the student answers discussed in this section are similar to those discussed in Sec. VII B above in that they are based on assumptions about the pressure at various points in the flow. Contrary to those, however, these students here apply intuitive rules for how the two quantities are related rather than using a memorized formula. Students in neither category use the principle of continuity.

\section{Inappropriate application of laws governing single particle motion}

Many of the students explained their answers by (implicitly) referring to the typical behavior of point particles under the influence of gravity (in IP) or friction (in HP and MP). We discuss the two types of reasoning separately in the two subsections below.

\section{Incorrect application of conservation of energy for a point particle}

About $15 \%$ of the first-year students after lecture explain their answer to question IP by arguing that a fluid "flowing uphill" slows down. This type of reasoning is illustrated by the following two examples.

IP: [less] Since position 2 is at a higher location than position 1. Therefore water flows more slowly at position 2. (Student 62)

IP: [less] Due to the incline of the pipe, the fluid will be slowed down. (Student 207)

Some of these students may base their answer on their everyday experience that a moving object without propulsion slows down while moving upward. Other students explicitly invoke the law of conservation of mechanical energy for a point particle, as the following quote illustrates ${ }^{5}$ :

IP: [less] At position 2 the potential energy is higher than at position $1 \rightarrow$ a fraction of the kinetic energy at position 1 will be converted to potential energy at position 2. (Student 349)

Arguments using potential and kinetic energy were significantly more prevalent in group 2 , with one in four students in this group reasoning in that way. However, we have no indication whether this type of thinking was enhanced by the teaching method, the lecturer, or the subjects addressed before.

In summary, we find that many students incorrectly generalize the behavior of point particles under the influence of gravity to fluids. This includes students who argue with the intuitive concept that an upward motion always decelerates, but also students who reason more formally with the law of conservation of mechanical energy for mass points. In the context of downward flows through vertical pipes, this type of student difficulty has been identified by Suarez et al. [9]. In our study, we have observed that students use the same kind of reasoning in other flows with changes in elevation. Moreover, as we discuss in the following section, similar types of reasoning that to our knowledge have not been documented in the literature become apparent in the context of friction as well.

\section{Belief of local kinetic energy loss due to friction}

Insight into students' thinking about the effect of friction on fluid flow can be gathered from their answers to questions HP (part b) and MP. In HP, after lecture instruction, $37 \%$ of the first-year students (groups 2 to 5) argue that the fluid is locally slowed down by friction. Often, the students support their answers by invoking energy arguments.

A typical example is this first-year student, whose answer to question IP was shown in Section VII D 1:

HP a): [same] no difference in elevation between 1 and 2, therefore kinetic energy remains the same (constant), $H P$ b): Yes [i.e., answer changes], since consequently a fraction of the kinetic energy is converted into heat with increasing distance. (Student 349)

As before (in IP), this student thinks of the relation of potential and kinetic energy for single particle motion. He reasons in HP (part a) that, as the height is not changing, the

\footnotetext{
${ }^{5}$ While this reasoning holds for point particles, fluid elements interact with each other, resulting in work being done by adjacent elements on each other and leading to the pressure term in the Bernoulli equation.
} 
potential energy is constant and therefore the kinetic energy is constant. When considering friction in HP (part b), he uses the correct argument that mechanical energy is converted to thermal energy, disregarding, however, that mass conservation imposes the condition that this conversion leads to a global, not local, reduction of the flow velocity: While in comparison of two different, but geometrically equal pipe systems the one with greater friction will result in lower flow velocity overall (and therefore smaller kinetic energy), this is not a valid argument for the comparison of two locations in one system.

Another student, also in Interior Engineering (group 2), makes assumptions about the pressure and uses a simplified Bernoulli principle (see Sec. VII B), thereby answering IP incorrectly and part a of HP correctly. The student then switches to an energy-based argument for part $b$ and states that kinetic energy is lost due to friction:

IP: [greater] since at this position (1), pressure is higher. HP a): [same] The pressure at 1 matches the one at 2. HP b): less than 1 (at 2), since due to friction on the pipe walls, kinetic energy gets lost. (Student 338)

Even students who in question IP and in part a of question HP argue correctly that the velocity is constant as a result of the uniform diameter, when prompted to consider friction (part b of HP) state that their answer would change. This answer pattern, given by more than $10 \%$ of the firstyear students after lecture (group 2 to 5), is illustrated by the following quote from a first-semester student in Wood Technology (group 4).

HP a): [same] Pipe diameter [is] constant, therefore also velocity [is] constant. HP b): Yes [i.e., the answer changes], with friction, the velocity at position 2 would be less than at position 1, since through friction, energy in the form of kinetic energy is lost. (Student 255)

Similarly to what has been observed in the context of elevation differences, this student apparently believes that due to the occurrence of friction, the previously used relation between pipe cross section and flow velocity (due to mass conservation) no longer holds. Some students state this idea even more explicitly as the following quote from another first-semester Wood Technology student indicates. This student's belief that friction slows things down seems to override his trust in the continuity equation.

IP: [same] The velocity depends on volume flow and the cross section of the pipe. Since both do not change, the velocity is the same at 1 and 2. HP a): [same] In an ideal flow (without friction) there are no losses. Moreover, the cross section and the volume flow are constant. $H P$ b): Yes [i.e., the answer changes], position 2 has a smaller velocity, since the velocity in the pipe decreases due to friction. (Student 276)
It is worth noting that this idea (i.e., that the continuity equation breaks down in the presence of friction) seems to occur even if friction is not mentioned explicitly in the problem statements (as is the case in MP). The following quote illustrates how a student who reasons correctly with the continuity equation in IP is distracted by the pressure drop that is indicated by the manometer readings in MP and concludes that the velocity at position 2 must be less than at position 1.

IP: [same] Continuity equation $\dot{V}=A \cdot v$. The volume flow is constant, and if the diameter remains constant, velocity must remain constant as well. MP a): [less] Due to laminar or turbulent flow a friction occurs, which leads to a pressure loss $\Delta p$. MP b): The water level is caused by the static pressure in the pipe. Due to friction a pressure loss occurs; therefore the water level decreases from tube to tube. (Student 59)

In summary, we note that many students' understanding of the continuity equation is not robust when friction needs to be considered. Single-particle type thinking, invoking energy or the effect of a frictional force, tends to mislead many students to disregard the continuity equation even if they are able to apply it correctly in situations they consider ideal.

\section{E. Difficulty interpreting the terms in Bernoulli's equation}

In this section, we present our interpretations of student answers that seem to indicate specific difficulties with the individual terms in the Bernoulli equation, i.e., with the concept of pressure and the kinetic and potential energy densities.

\section{General difficulties with the pressure concept}

Previous studies have indicated that during their first exposure to hydrostatics, students often have severe difficulties understanding the concept of pressure [23]. Among others, this includes the difficulty to interpret pressure as describing a point in space rather than a property of an object; a failure to distinguish between pressure and force; and the idea that pressure is associated with a direction. In our investigation, we observed similar difficulties, as, for example, the idea that pressure is distributed over a region, that there are multiple, opposing pressures acting at one point, or that pressure decreases with distance from a reservoir. We will not illustrate or discuss these in detail.

Specifically in the context chosen here, the role and consequences of the manometer tubes shown in MP seem to be problematic for some students. One student seemed to believe that the presence of the water columns acted as an accelerating agent on the water in the horizontal pipe, 
MP a): [same] Due to the gravitational pressure of the individual columns, the water in the pipe experiences an accelerating pressure. (Student 302)

aaawhile several others seemed to think that the openings of the tubes at the top result in a decrease of the velocity:

MP a): [less] Because the water can escape, the pressure decreases, and therefore also the flow velocity. (Student 329)

$M P$ a): [less] Since the flow loses velocity as a result of the water flowing into the tubes. (Student 347)

One student associated changes in pressure with the openings of the tubes while asserting that in a tube without such openings, even if inclined (as in IP), the pressure must be equal everywhere:

IP: [same] Since the water pressure is the same everywhere. MP a): [less] Water pressure decreases through the openings; therefore different water levels in the tubes. (Student 317)

We speculate that for some students, the idea of pressure implies a pressure that is greater than atmospheric pressure and is closely linked with the condition of confinement or a material barrier between the fluid under pressure and its surroundings. The latter interpretation is consistent with results seen in the context of hydrostatics [23].

\section{Failure to consider all relevant terms in Bernoulli's equation}

In their answers, some students quoted in Sec. VII B (students 257, 351,37, and 208) omit terms of the Bernoulli equation, thereby using a simplified relation between pressure and velocity while skipping other terms like the potential energy, the static pressure or the pressure loss. (This has been observed by Suarez et al. in the case of the static pressure or the potential energy in the context of their question 1 [9].) We suppose that the students tendency to leave out certain terms may result from their difficulty to associate meaning with these terms. Moreover, these students do not realize that the problem cannot be solved with their approach, suggesting additional difficulties with multiple-variable equations (see Sec. VII H 1).

\section{Lack of distinction between hydrostatic pressure and potential energy per unit volume}

Bernoulli's equation is often presented in a form such that it relates three (or more) terms all having the dimension of pressure. Sometimes (and quite frequently in German textbooks on hydrodynamics) these quantities are given specific names such as static, dynamic, and geodesic pressure which, predictably, lead to confusion. Moreover, the term $\rho g h$ (with $h$ increasing upward) in
Bernoulli's equation usually denotes the potential energy per unit volume whereas it is often used in hydrostatics to describe the altitude-dependent term in the static pressure or hydrostatic pressure (with $h$ increasing downward). This variation of static pressure with altitude is only implicit in Bernoulli's equation.

We observed in numerous answers that students interpret the term $\rho g h$ incorrectly (see, for example, students 133 and 257 whose answers were discussed in Sec. VII B). By referring to it as hydrostatic pressure or static pressure, students seem to imply that it is the only altitude-dependent pressure term in Bernoulli's equation, i.e., that $p$ may not (even implicitly) depend on altitude.

\section{F. Student difficulties with viscosity, fluid friction, and the velocity profile}

In the context of the three questions that we posed, we observed some other student difficulties that pertain to other properties of fluid flow that are not completely described by the continuity or Bernoulli's equations.

\section{Belief that friction only occurs between pipe walls and outer fluid layer}

In their answers to HP (part b) and MP, students displayed a wide variety of correct and incorrect ideas about friction in fluids. A frequently mentioned incorrect belief is that friction occurs only between the outermost fluid layer and the pipe wall. For example, student 338, who was quoted in Sec. VII D 2, seems to visualize friction in a fluid context just like the (dry-dry) friction occurring between two solid objects, thereby concluding that the water flows more slowly at position 2 as compared to position 1.

For other students, this type of thinking leads to a correct answer to HP (part b), as friction does not affect the velocity of the fluid in the center of the pipe. The following quotes from first-semester students in Wood Technology and Interior Engineering (after lecture and laboratory or only lecture, respectively) illustrate this:

$H P(b)$ : No [i.e., the answer does not change], friction only occurs between the surface layers of the water and the pipe wall, not in the middle layer. (Student 228)

$H P$ b): No [i.e., the answer does not change], since the flow is measured in the center of the pipe; not at the boundary where the flow [sic] occurs. (Student 346)

$H P b$ ): The flow in the center remains constant; a flow profile develops in the pipe. (Student 345)

To illustrate their argument, the last one of the three students quoted here even included a sketch of a parabolic flow profile with their answer. 


\section{Belief that only spatial variation in friction matters}

In their answers to part b of question HP (asking whether their answer to part a would change if friction were considered), several students in the Energy and Building Technology groups before or after lecture (i.e., groups 1 or 5) argued that friction would not affect their answer as long as it is the same at both positions.

HP b): No, because the roughness of the pipe can be neglected, because it is the same everywhere. (Student 67)

$H P$ b): No, it does not change since the resistance is constant everywhere and therefore acts "equally" on the whole system. (Student 23)

One student explicitly adds that a spatial variation in friction would lead to a different answer.

IP: [same] Because the pipe diameter remains the same, the velocity is constantly equal. HP a): [same] Here, too, the pipe diameter is the same, and therefore the velocity also remains the same. $H P$ b) If the friction is the same at 1 and 2, then the velocity remains the same as well. If it [the friction] were different, it [the velocity] would change... (Student 68)

In particular student 68 seems to treat friction on the same footing as pipe diameter, as if the local value of this quantity were related to the local value of the velocity.

\section{G. A brief comment regarding compressibility}

Brown et al. [8] and Suarez et al. [9] both report that students frequently conceptualize water as a compressible fluid. Brown et al. for example, find statements that they attribute to the concept of compressibility in between onethird and one-half of their interviews, depending on the population. In the data obtained through our test, we do not see any evidence for the hypothesis that students think of water as a compressible fluid. This may be due to the fact that the questions we posed do not involve a narrowing or widening of the cross section of a pipe (as in the case of both other publications). Moreover, we did not explicitly ask students about the pressure in any of the three questions IP, HP, and MP.

However, in situations in which a change in pressure can be inferred (e.g., in question MP) there is no indication that students conceive of the water as changing its density. Consequently, we hesitate to interpret some of the codes listed in Ref. [8] (as, for example, "water compresses") as implying an increase or decrease in the density of water. "Compress" (in active or passive voice) as used by the students (e.g., student 107 in that article) may simply express the notion of being under pressure rather than occupying a smaller volume.

\section{H. General difficulties in describing and explaining scientific and technical situations}

Some student responses to the questions we asked can be interpreted as reflecting more general reasoning difficulties that seem independent of the particular quantities describing fluid flow and may therefore also arise in the context of other subject matter. We discuss these separately in this section, noting in each case if these or similar difficulties have indeed been observed in other contexts in introductory physics or engineering.

\section{Difficulties with equations containing multiple variables}

As has been observed by Brown et al. many students explain changes in one quantity in terms of a single other variable, for example, by relating pressure or velocity only to the cross-sectional area of the pipe, as if there were no other relevant quantities that could change [8]. Some of the answers to question IP that we presented in Sec. VII B display the same reasoning difficulty, as one may only obtain a (perceived) answer to this question through Bernoulli's law by ignoring one other term (most commonly the potential energy density) in that equation.

Among the answers to the same question, we have found one other difficulty that seems related: the conclusion that in a sum of three terms with constant value, if one of the terms increases and one other decreases, the third must stay the same. The following quote from a first-semester Wood Technology student (group 4) illustrates this type of reasoning.

$$
\begin{aligned}
& I P:[\text { same }]: p_{\text {stat } 1}+p_{\text {dyn } 1}+p_{\text {geo } 1}=p_{\text {stat } 2}+p_{\text {dyn } 2}+p_{\text {geo } 2} ; \\
& p_{\text {geo } 1}<p_{\text {geo } 2} ; p_{\text {stat } 1}>p_{\text {stat } 2} \Rightarrow p_{\text {dyn } 1}=p_{\text {dyn2 }} \text { (Student } \\
& \text { 250) }
\end{aligned}
$$

This student argues with a complete Bernoulli equation and arrives at a correct answer, though with faulty reasoning, by implicitly assuming that the static pressure decreases by the same amount as the potential energy density (here denoted as the geodesic pressure) increases. While the two amounts are indeed equal, the assumption is still invalid as the student fails to recognize that in the given situation the continuity equation imposes a uniform flow velocity throughout the pipe which, in turn, leads to the assumed relationship of the two terms in question.

The reasoning shown above is, of course, equivalent to concluding that the sum of the two terms must stay the same. We note that a similar type of reasoning, namely, that an increase in one quantity and a decrease in another imply that their product is constant, has been observed previously in the context of the ideal gas law [25]. We assume that both types of reasoning stem from a common notion that opposite changes of contributions or "factors" in a complex phenomenon tend to cancel each other out. This underlying 
notion may be characterized as a phenomenological primitive similar to the $p$-prims in the category "balance and equilibrium" identified by diSessa in Ref. [26].

The attractiveness to student thinking of an assumed inverse proportionality also becomes evident from the fact that for the reasoning about question HP we occasionally saw "Bernoulli's equation" written as $p_{1} v_{1}=p_{2} v_{2}$.

\section{Failure to distinguish spatial differences from changes with respect to time}

Several students, mostly (but not only) among those with self-study and before lecture instruction (group 1), explained their correct answers to IP and HP or MP by referring to the constancy of the water level in the container or in the manometer tubes. To illustrate this type of reasoning, we present answers to the two versions of the test, both from students in Energy and Building Technology:

IP: [same] Since for the whole time the water level is kept at the same height by the refill mechanism. HP a): [same] Same phenomenon as in (1). The water level in the container is kept at the same height for the whole time by a refill mechanism. (Student 23)

IP: [same] The water level remains constant $\Rightarrow U n i-$ form pressure. HP a): [same] Since the levels are always constant, the pressure is also always constant. $\Rightarrow$ constant velocity. (Student 16)

In both their answers, the students use the constancy of one quantity with respect to time to infer that another quantity has the same value at two different points. That these are different comparisons seems to be difficult to recognize for some students being exposed to physics at the post-secondary level for the first time. Informal use of "constant" for time and spatial dependencies, even by instructors, may worsen the situation.

\section{Difficulty recognizing the relevance of idealizations}

In Sec. VII D 2, we have discussed the frequently stated answer to question HP that in the presence of friction, the flow velocity will not be the same at the two positions in the pipe. The reasoning given by students often invoked the loss of kinetic energy or the general tendency of friction to slow objects down. Above, we interpreted this type of reasoning as an inappropriate extension of ideas about single-particle motion to the behavior of fluids, i.e., a failure to recognize that the object in question is the entire fluid body and that the rules governing its behavior (e.g., the continuity equation) must still hold.

More broadly, this difficulty can be seen as a failure to recognize the validity of general laws of nature and the relevance of certain idealizations, leading students to incorrectly discard fundamental laws of nature in the presence of nonideal conditions (i.e., as ideal approximations are relaxed). In this case, students are willing "to give up" the continuity equation (and thereby conservation of mass) in the presence of friction. An analoguous difficulty has been observed in the context of electric circuits by McDermott and Shaffer [10] (among others). While in that context, there is no transition from an ideal to a nonideal model (since electric circuits without resistive elements would be considered pointless), students still predicted unequal currents "before" and "after" a resistive element (light bulb) due to that bulb's resistance. An example of a similar difficulty from a different context (where both ideal and nonideal models are considered) is given by Kautz and Schmitz, who report that more than a third of the students in an introductory thermodynamics course claim that the entropy of a system increases over the course of a complete cycle in an arbitrary cyclic process when dissipative effects are taken into account, regardless of the entropy being a state function [27].

\section{Uncertainty in the prioritization of concepts in a given situation}

Many of the students in the classes we surveyed seem to have difficulty in deciding which physical principles to use in solving the problem we posed to them. This uncertainty becomes most evident either when students change their initial answers (and may even comment on this change) or when they use different strategies for the same problem in the case of different boundary conditions. Examples of the former type of answer are the following, given by two students, second- and first-year, respectively, whose ideas about the pressure in the pipe are clearly interfering with their original idea that the answer is determined by the geometry of the pipe:

IP: [same] Since the diameter is the same!

[less] Because of the pressure difference! But the diameter stays the same, therefore I am unsure. (Student 104)

IP: [same]

[less] $\dot{V}=A \cdot v \rightarrow$ constant cross section $\rightarrow$ unchanged volume flow, but influence of hydrostatic pressure and changed location. (Student 300)

An example of the latter type of answer is the following, where a first-semester student in Interior Engineering (after lecture) uses three different approaches to answer all three tasks:

IP: [less] static pressure increases, geodesic pressure [potential energy density] remains the same $\rightarrow$ dynamic pressure decreases. HP a): [same]

$A_{1} \cdot \bar{v}_{1}=V[\mathrm{sic}]=A_{2} \cdot \bar{v}_{2} ; A_{1}=A_{2} \Rightarrow v_{1}=v_{2}$ since $V$ is constant. HP b) Yes [i.e., the answer changes], friction works against $V \rightarrow v$ decreases; $v($ pos.2) $<$ $v$ (pos.1). (Student 319) 
In answering question IP, the student starts with (incorrect) assumptions about two of the terms in Bernoulli's equation and argues in the manner discussed in Sec. VII B. When addressing HP (part a), however, the student changes strategy and correctly uses the continuity equation. Finally, for HP b), the student argues based on friction (as discussed in Sec. VII D 2), not realizing that the answers to the three tasks are inconsistent. As the following student quote indicates, a change in strategy may also occur in the opposite direction (i.e., starting with the correct approach in IP and then using incorrect reasoning based on Bernoulli's equation):

IP: [same] velocity [is] constant, as volume flow and diameter of pipe [are] constant, $\dot{V}=A \cdot \bar{v} . \quad M P$ : [greater] As the pressure at 2 has dropped, the velocity must have increased. (Student 57)

Student reasoning in this context is, in some way, analogous to behavior seen in the context of kinematics. There, as Shaffer and McDermott observed [28], students often attempt to solve kinematics tasks by invoking Newton's second law, thereby relating the acceleration to an assumed (net) force even if insufficient information is given to solve the problem in this way. We believe that it is not accidental that in both situations students apply laws that relate the motion of objects (or fluids) to the perceived cause (force or pressure) of that motion, rather than to relationships that simply serve to generate a consistent description of this motion.

\section{Tendency to reason locally or sequentially rather than holistically}

In the context of electric circuits, McDermott and Shaffer have observed that "there is a tendency to think locally or sequentially, rather than to reason holistically. Students may think of a circuit [...] as consisting of components that can be analyzed one after another in sequence around the circuit" [10]. While the tasks we posed did not involve an explicit change made at a single point in the pipe system, we believe that similar kind of reasoning underlies many of the students' incorrect answers in our study. By comparing the inclined pipe to a level pipe, students incorrectly conclude that the inclination of the pipe affects the flow only downstream, thereby resulting in an assumed difference in flow velocity at the two positions. Similarly, comparing the flow with friction to the frictionless case, students argue in an analogous way. The reasoning by student 68 discussed in Sec. VII F 2, that the amount of friction at a particular position in the flow affects the flow velocity at that point, can be interpreted as a particular case of such local reasoning. In the introductory physics laboratory course, we observe similar reasoning also in problems concerning the heat transfer through a series of building components, suggesting that student thinking about flows of incompressible fluids, electric charge. and thermal energy is, to some extent, affected by similar conceptual difficulties.

\section{IMPLICATIONS FOR TEACHING FLUID MECHANICS}

The results presented here as well as those from other studies on student understanding of fluid mechanics $[8,9]$ indicate that many students do not develop a functional understanding of the essential concepts of this topic as a result of standard instruction. Based on this conclusion, Brown et al. suggest using "an active learning approach to encourage conceptual change." Moreover, "students should be encouraged to create, defend, and test hypotheses with classmates to evaluate their beliefs and understanding of fluid mechanics [...]."

The four groups of first year students in our study who had completed the unit on fluid mechanics differed with respect to the format of instruction and therefore allow us to test the suggestion made by Brown et al. More specifically, groups 2 and 3 received traditional lectures with or without laboratory whereas groups 4 and 5 were taught through active learning methods such as Just-in-Time Teaching and Peer Instruction, in addition to the laboratory.

In the Just-in-Time Teaching format [21], students are given a weekly study assignment (including textbook reading, answering online pretest questions and posing their own questions about the content) to prepare for the upcoming class session. The instructor then uses this student input to design the class activities "just in time." This practice is often combined with Peer Instruction [22], which is a three-step procedure. Students first answer conceptual multiple-choice questions individually and then discuss them with their neighbors. Finally, the instructor conducts a class discussion on various types of reasoning that lead to correct and incorrect answers.

Consistent with Brown's expectation the percentage of correct answers with correct reasoning were generally higher in the two groups taught with PI or JiTT than in the traditionally taught groups:

- IP: $50 \%$ and $62 \%$ (JiTT/PI) versus $23 \%$ and $44 \%$ (traditional)

- HP: $35 \%$ and $45 \%$ (JiTT/PI) versus $18 \%$ and $6 \%$ (traditional)

- MP: $73 \%$ and 53\% (JiTT/PI) versus $16 \%$ and $27 \%$ (traditional)

as illustrated in Fig. 2 above.

Combining the two groups undergoing similar instruction (group 4 and 5, and group 2 and 3, respectively) in each comparison, we have checked for significance using the chi-square test. The resulting probabilities for differences this large or greater were calculated to be less than 0.01 for all three questions, i.e., the differences are highly significant. These results indicate that active learning methods have a much better chance of helping students develop and apply a conceptual understanding of the subject matter. In part, this may be due to strengthened reasoning abilities. This interpretation is consistent with results from other studies [29,30]. 
The active-learning methods (JiTT and PI) used in groups 4 and 5 within our study can be seen as a first step to implement these suggestions and do show some improvement over traditional lecture instruction (see Sec. VIII). Even in these groups, however, the percentage of correct answers and reasoning for one of the questions on the test does not exceed $45 \%$, indicating that even with the methods used here, results are not fully satisfying.

Taking the idea of conceptual change one step further, we posit that students need to become aware of their own initial conceptions and recognize the need to extend or modify them. This approach has been described by McDermott with the phrase "elicit, confront, resolve" [31], suggesting that students (i) be led to make a prediction about a physical situation, (ii) be faced with evidence that may contradict their expectation, and (iii) be supported in resolving that conflict through a step-by-step development of a correct model. Qualitative collaborative-group worksheets, as, for example, the "Tutorials in Introductory Physics" [32], may constitute a concrete realization of this approach for the practice of instruction. They are often preceded by a pretest that is part of the instructional process (elicit) and may be used for purposes of assessment. Following this same path, we began the development of a set of tutorials for fluid mechanics, which we describe in a forthcoming publication. The results of the present paper indicate that this effort has to begin with the topic of the continuity equation. Specifically, students need help in developing a model for mass conservation in fluid flow that is robust against "disturbing factors" such as the influence of gravity, dissipation, or changes in geometry.

\section{SUMMARY AND CONCLUSIONS}

In this study, we investigated student understanding of the continuity principle in fluid mechanics among students in various engineering bachelor programs at a University of Applied Sciences in Germany.

To probe student thinking, we used multiple-choice questions with open-ended written explanations and analyzed student answers both quantitatively and qualitatively. Through a multistep coding process, we identified characteristic patterns of student reasoning.

We found that students have difficulty applying and prioritizing the two basic principles of mass conservation (continuity equation) and energy conservation (Bernoulli's equation) when distracted by questions that involve gravity, dissipative effects or a visible pressure drop. Among firstyear students, the maximum percentages of students answering correctly were $62 \%, 45 \%$, and $73 \%$, respectively, in the three situations. Instead of invoking the continuity equation, a considerable number of students based their answers on ill-supported assumptions about local pressures and then either applied Bernoulli's law ("since the pressure drops, according to Bernoulli the velocity must increase") or a naïve association between the two quantities ("higher pressure leads to higher velocity"). Others based their reasoning on analogies to singleparticle motion ("flow velocity decreases when flowing upwards or friction is present").

More generally, our data indicate that students have difficulties in realizing the meaning of idealizations, recognizing the hierarchy of different principles, applying equations containing multiple variables, and understanding the cumulative effect of friction regarding dissipative losses. We did not, however, see any evidence of students thinking of water as a compressible fluid. This result suggests that any difficulties students may have with applying the continuity equation do not primarily stem from a failure to assume water to be (nearly) incompressible.

Among the different groups of students in the study, we observed differences in success rates that correlated with the respective methods of instruction. Groups taught using active-learning formats such as Peer Instruction or Just-inTime Teaching achieved substantially better results than those taught traditionally. Still, using performance on the question involving friction (HP) as a criterion, even in these groups at least one-half of the students failed to develop a functional understanding of continuity. This result demonstrates the need for the development of instructional materials that takes into account student difficulties.

One of the limitations of our study is the fact that we only surveyed students from a single university that draws from a particular sector of the student population in Germany. However, investigations of student understanding of other topics in the introductory physics curriculum showed qualitatively similar results across different countries and types of universities (even if the percentage of specific patterns of thinking may differ). This is true in particular for student difficulties in the context of electric circuits, which in some respect are mirrored by our results. We therefore expect that students in engineering programs at other universities in Germany and other countries will display similar types of difficulties.

Finally, we want to point out that our conclusions about more general reasoning difficulties that students may have are still somewhat hypothetical. As indicated above, there exist results from other branches of physics that seem to follow similar patterns. However, more research involving a wide range of topics needs to be carried out (or results of existing work need to be revisited) to allow more definite conclusions about such general reasoning difficulties.

\section{ACKNOWLEDGMENTS}

We acknowledge funding from the STEM-programs "MINTerAKTIV/BayernMINT" of the Bavarian Ministry of Science and Art. 


\section{APPENDIX: QUIZ ON FLUID FLOW}

We provide an English translation of the three questions that were used in the short written test.

Question 1 Inclined-Pipe question (IP): Water continuously flows out of a wide, water-filled tank through an inclined pipe with uniform diameter [see Fig. 1 on the left]. The water level of the tank is kept constant by a refill mechanism. Consider the water flowing in the pipe.

Is the velocity of the water in the middle of the pipe at position 2

$\square$ greater than

less than

$\square$ the same as

the velocity at position 1? Please explain why this answer is correct.

Question 2 Horizontal-Pipe question (HP): Water continuously flows out of a wide, water-filled tank through a horizontal pipe with uniform diameter [see Fig. 1 in the middle]. The water level of the tank is kept constant by a refill mechanism. Consider the water flowing in the pipe. Assume that friction can be neglected.

a) Is the velocity of the water in the middle of the pipe at position 2 $\square$ greater than

$\square$ less than

the same as

the velocity at position 1? Please explain why this answer is correct.

b) Would your answer change if friction were to be taken into account? Please explain why.

Question 3 Manometer-Pipe question (MP): Water continuously flows out of a wide, water-filled tank through a horizontal pipe with constant diameter [see Fig. 1 on the right]. The water level of the tank is kept constant by a refill mechanism. The water level of the three manometer tubes is also constant. Consider the water flowing in the pipe.

a) Is the velocity of the water in the middle of the pipe at position 2

$\square$ greater than

$\square$ less than

$\square$ the same as

the velocity at position 1? Please explain why this answer is correct.

b) Please also explain, if you have not yet done so, the water levels in the manometer tubes.
[1] B.E. Faulkner and F. M. Ytreberg, Understanding Bernoulli's principle through simulations, Am. J. Phys. 79, 214 (2011).

[2] A. Rahman, A blended learning approach to teach fluid mechanics in engineering, Eur. J. Eng. Educ. 42, 252 (2017).

[3] A. Samsudin, N. Fratiwi, N. Amin, Wiendartun, Supriyatman, F. Wibowo, M. Faizin, and B. Costu, Improving students' conceptions on fluid dynamics through peer teaching model with PDEODE (PTMPDEODE), J. Phys. Conf. Ser. 1013, 012040 (2018).

[4] J. Martin, J. Mitchell, and T. Newell, Development of a concept inventory for fluid mechanics, in Proceedings of the 33rd Annual Frontiers in Education (FIE) Conference 2003, T3D-23 Westminster Colorado (IEEE, Stipes Publishing L.L.C, Champaign, 2003).

[5] J. K. Martin, J. Mitchell, and T. Newell, Work in progress: Analysis of reliability of the fluid mechanics concept inventory, in Proceedings of the 34th Annual Frontiers in Education (FIE) Conference 2004, F1F-3 Savannah (IEEE, Bellingham, WA, 2004).

[6] B. M. Olds, R. A. Streveler, R. L. Miller, and M. A. Nelson, Preliminary results from the development of a concept inventory in thermal and transport science, in Proceedings of the 2004 ASEE Annual Conference and Exposition, 9.1003.1 Salt Lake City (ASEE, Washington D.C., 2004).

[7] M. Watson, A. Mills, K. Bower, K. Brannan, M. Woo, and R. Welch, Refinement of a concept inventory to assess conceptual understanding in civil engineering fluid mechanics, in Proceedings of the 2015 ASEE Annual Conference and Exposition, 26.1322.1 Seattle (ASEE, Washington D.C., 2015).

[8] S. A. Brown, K. Beddoes, D. Montfort, and A. Baghdanov, Engineering students fluid mechanics misconceptions: A description and theoretical explanation, Int. J. Eng. Educ. 33, 1149 (2017).

[9] A. Suarez, S. Kahan, G. Zavala, and A. C. Marti, Students conceptual difficulties in hydrodynamics, Phys. Rev. Phys. Educ. Res. 13, 020132 (2017).

[10] L. C. McDermott and P. S. Shaffer, Research as a guide for curriculum development: An example from introductory electricity. Part i: Investigation of student understanding, Am. J. Phys. 60, 994 (1992).

[11] C. Schäfle and C. Kautz, Students' reasoning in fluid dynamics: Bernoulli's principle vs the continuity equation, in Proceedings of the 10th International Conference on Physics Teaching in Engineering Education, Delft, The Netherlands 2019 (The Hague University of Applied Sciences, Delft) (to be published).

[12] C. Schäfle and C. Kautz, Students' reasoning in fluid dynamics: Bernoulli's principle vs the continuity equation, contributed poster, in Proceedings of the Physics Education Research Conference, Washington D.C. (AIP, New York, 2018).

[13] H. Pfundt and R. Duit, Students' alternative frameworks and science education: Bibliography, available at 
https://archiv.ipn.uni-kiel.de/stcse, Institute for Science Education at the University of Kiel, Germany (1994).

[14] D. Hammer, Student resources for learning introductory physics, Am. J. Phys. 68, S52 (2000).

[15] A. A. DiSessa, Towards an epistemology of physics, Cognit. Instr. 10, 105 (1993).

[16] J. Minstrell, Facets of students' knowledge and relevant instruction, in Research in physics learning: Theoretical issues and empirical studies, in Proceedings of an International Workshop, edited by R. Duit, F. Goldberg, and H. Niedderer (Institute for Science Education, Germany, 1992).

[17] P. R. Heron, Empirical investigations of learning and teaching, Part I: Examining and interpreting student thinking, in Proceedings of the International School of Physics Enrico Fermi Vol. 156 (IOS Press, Amsterdam, NL, 2004), p. 341.

[18] L. C. McDermott, Oersted Medal Lecture 2001: Physics education research: The key to student learning, Am. J. Phys. 69, 1127 (2001).

[19] P. A. Tipler and G. Mosca, Physics for Scientists and Engineers (W.H. Freeman, San Francisco, CA, 2008), p. 443.

[20] D. Hestenes, M. Wells, and G. Swackhammer, Force Concept Inventory, Phys. Teach. 30, 141 (1992).

[21] G. M. Novak, E. T. Patterson, A. D. Gavrin, and W. Christian, Just-in-Time Teaching: Blending Active Learning with Web Technology (Prentice Hall, Englewood Cliffs, NJ, 1999).

[22] E. Mazur, Peer instruction: A user's manual (Prentice Hall, Englewood Cliffs, NJ, 1997).

[23] M. Loverude, P. Heron, and C. Kautz, Identifying and addressing student difficulties with hydrostatic pressure, Am. J. Phys. 78, 75 (2010).
[24] M. T. Chi, J. D. Slotta, and N. De Leeuw, From things to processes: A theory of conceptual change for learning science concepts, Learn. Instr. 4, 27 (1994).

[25] C. H. Kautz, P. R. Heron, M.E. Loverude, and L. C. McDermott, Student understanding of the ideal gas law, Part i: A macroscopic perspective, Am. J. Phys. 73, 1055 (2005).

[26] A. A. DiSessa, Why conceptual ecology is a good idea, in Reconsidering conceptual change: Issues in Theory and Practice (Springer, New York, 2002), p. 28.

[27] C. H. Kautz and G. Schmitz, Probing student understanding of basic concepts and principles in introductory engineering thermodynamics, in ASME 2007 International Mechanical Engineering Congress and Exposition (American Society of Mechanical Engineers Digital Collection, New York, 2008), p. 473.

[28] P. S. Shaffer and L. C. McDermott, A research-based approach to improving student understanding of the vector nature of kinematical concepts, Am. J. Phys. 73, 921 (2005).

[29] R. R. Hake, Interactive-engagement versus traditional methods: A six-thousand-student survey of mechanics test data for introductory physics courses, Am. J. Phys. 66, 64 (1998).

[30] S. Freeman, S. L. Eddy, M. McDonough, M. K. Smith, N. Okoroafor, H. Jordt, and M. P. Wenderoth, Active learning increases student performance in science, engineering, and mathematics, Proc. Natl. Acad. Sci. U.S.A. 111, 8410 (2014).

[31] L. C. McDermott, Millikan lecture 1990: What we teach and what is learned: Closing the gap, Am. J. Phys. 59, 301 (1991).

[32] L. C. McDermott, P. S. Shaffer, and Physics Education Group, Tutorials in Introductory Physics, prelim. ed. (Prentice Hall, Upper Saddle River, NJ, 1997). 